Comment. Math. Helv. 74 (1999) 398-418

0010-2571/99/030398-21 \$1.50+0.20/0
(C) 1999 Birkhäuser Verlag, Basel

Commentarii Mathematici Helvetici

\title{
Concentration multi-échelles de courbure dans des fibres de Milnor
}

\author{
Evelia García Barroso et Bernard Teissier
}

Résumé. Etant donné un germe de morphisme analytique complexe $f:\left(\mathbf{C}^{2}, 0\right) \rightarrow(\mathbf{C}, 0)$ à fibre réduite, nous étudions la manière dont la courbure de Lipschitz-Killing de la fibre de Milnor $C(\lambda)_{\epsilon}=f^{-1}(\lambda) \cap \mathbf{B}_{\epsilon} \subset \mathbf{C}^{2}$ pour la métrique induite par celle de $\mathbf{C}^{2}$ se concentre asymptotiquement, lorsque $(\epsilon, \lambda) \rightarrow(0,0)$, dans l'intersection de cette fibre avec des boules dont les centres $\xi_{q, l}^{Q}(\lambda)$ peuvent être décrits mais surtout dont les rayons sont de la forme $|\lambda|^{\rho(Q)}$ où les $\rho(Q)$ sont des nombres rationnels dont la collection ne dépend que de la topologie du plongement dans $\mathbf{C}^{2}$ du germe de courbe plane réduite $C=C(0)$.

\begin{abstract}
Given a complex-analytic map-germ $f:\left(\mathbf{C}^{2}, 0\right) \rightarrow(\mathbf{C}, 0)$ with reduced fiber, we study how the Lipschitz-Killing curvature of the Milnor fiber $C(\lambda)_{\epsilon}=f^{-1}(\lambda) \cap \mathbf{B}_{\epsilon} \subset \mathbf{C}^{2}$ for the metric induced by that of $\mathbf{C}^{2}$ concentrates asymptotically, as $(\epsilon, \lambda) \rightarrow(0,0)$, in the intersection of this fiber with balls, whose centers $\xi_{q, l}^{Q}(\lambda)$ can be described, but whose radii, more importantly, are of the form $|\lambda|^{\rho(Q)}$ where the $\rho(Q)$ are rational numbers depending only on the topological type of the embedding in $\mathbf{C}^{2}$ of the germ of reduced plane curve $C=C(0)$.
\end{abstract}

Mathematics Subject Classification (1991). 32S55.

Mots-clès. Singularités, Courbe plane, Fibration de Milnor, Courbure.

\section{Introduction}

Dans ce travail nous étudions le comportement asymptotique de la courbure de la fibre de Milnor

$$
C(\lambda)_{\epsilon}=f^{-1}(\lambda) \cap \mathbf{B}_{\epsilon} \subset \mathbf{C}^{2}
$$

d'un germe de courbe plane réduite $(C, 0) \subset\left(\mathbf{C}^{2}, 0\right)$ défini par une équation $f(x, y)=0$ lorsque $\epsilon$ et $\lambda$ tendent vers 0 . Il s'agit de la courbure de LipschitzKilling associée à la métrique induite sur $C(\lambda)$ par celle de $\mathbf{C}^{2}$. On connait déjà, grâce au travail de Langevin [La] la valeur limite de l'intégrale de cette courbure:

$$
\operatorname{Lim}_{\epsilon, \lambda \rightarrow 0,|\lambda|<<\epsilon} \int_{C(\lambda)_{\epsilon}}|K| d v=2 \pi\left(\mu^{(2)}(C)+\mu^{(1)}(C)\right),
$$


où $\mu^{(2)}$ est le nombre de Milnor en 0 de la singularité $C(0)=C$ et $\mu^{(1)}$ sa multiplicité en 0 diminuée de 1 . Il faut souligner que le terme de droite ne dépend que de la topologie du plongement dans $\mathbf{C}^{2}$ du germe de courbe plane réduite $(C, 0)$. D'après [Te3], le nombre $\mu^{(2)}(C)+\mu^{(1)}(C)$ est le nombre d'intersection à l'origine de $C$ avec une de ses courbes polaires relatives génériques, qui sont définies par les équations

$$
\frac{\partial f}{\partial y}+\tau \frac{\partial f}{\partial x}=0
$$

C'est aussi le nombre des points d'intersection (transverses) d'une telle courbe polaire avec une fibre de Milnor $f(x, y)-\lambda=0$ qui tendent vers 0 avec $\lambda$. Ce résultat est donc au fond de la nature d'un résultat de théorie de l'intersection, c'est à dire que l'on compte des points, ou le degré de cycles, sans se préoccuper de leur position.

Nous allons obtenir une information plus précise sur la géométrie de la fibre de Milnor en essayant de localiser les régions de $C(\lambda)$ où se concentre asymptotiquement la courbure, et ce faisant mettre en évidence le fait que la concentration de courbure est un phénomène multi-échelles: la courbure se concentre (voir le corollaire 3.1) dans les intersections avec $C(\lambda)$ de boules, dont les centres $\xi_{q, l}^{Q}(\lambda)$ peuvent être décrits, mais surtout dont les rayons sont de la forme $|\lambda|^{\rho(Q)}$ où les $\rho(Q)$ sont des nombres rationnels dont la collection ne dépend que de la topologie du plongement dans $\mathbf{C}^{2}$ du germe de courbe plane réduite $(C, 0)$. Il nous semble intéressant que cette description multi-échelles elle même ne dépende que de la topologie.

Lorsque le germe $C$ est analytiquement irréductible en 0, i.e., est une branche, la donnée des exposants $\rho(Q)$, qui sont alors en nombre égal à celui des exposants de Puiseux, et celle de la quantité de courbure qui se concentre dans les boules de rayon $\rho(Q)$ permettent de déterminer les exposants caractéristiques de Puiseux de $C$, et l'on peut donc dire que le comportement asymptotique de la courbure de la fibre de Milnor permet de déterminer la classe d'équisingularité de la fibre limite singulière. Le cas réductible est plus compliqué, en particulier parce qu'une partie de la courbure reste "diffuse", comme dans le cas extrême de $x^{n}+y^{n}=0$, où il n'y a pas de concentration de courbure dans des boules de centre différent de l'origine (et donc dépendant de $\lambda$ ). Nous savons mesurer quelle est la partie diffuse.

Une autre manière de décrire ce phénomène de concentration est de dire qu'il exprime en partie comment la structure du diviseur exceptionnel $E$ d'une résolution des singularités plongée minimale $(Z, E) \rightarrow\left(\mathbf{C}^{2}, 0\right)$ de $(C, 0)$ se réflète dans la géométrie de la fibre de Milnor. Le caractère multi-échelles provient, de ce point de vue, de la structure non-linéaire du graphe d'intersection de ce diviseur exceptionnel, c'est à dire de l'existence de "sommets de rupture" dans ce graphe, selon une correspondance étudiée en détail dans [GB1], et il serait intéressant de donner une preuve de notre résultat fondée sur le relèvement à la surface $Z$ où $C$ est résolue de la métrique de $\mathbf{C}^{2}$ en une métrique singulière le long du diviseur exceptionnel. 
Notre technique de preuve est toute différente et est basée sur l'analyse du contact (voir le $\S 1$ pour une définition précise) avec les branches de $C$ des différentes branches des courbes polaires génériques qui a été faite dans [GB1], [GB2].

L'idée heuristique part du fait que par définition de la courbe polaire $\frac{\partial f}{\partial y}+\tau \frac{\partial f}{\partial x}=$ 0 , ses points d'intersection avec $C(\lambda)$ sont les points de $C(\lambda)$ où la tangente a la direction correspondant au paramètre $\tau$. Pour prouver le théorème de Langevin, on compte le nombre de ces points et on applique la formule d'échange (Théorème 3.1). Nous observons que ces points ont répartis sur les différentes branches de la courbe polaire, et si le contact avec $C$ en 0 d'une de ces branches est fort, elle varie peu lorsque l'on varie le paramètre $\tau$ et par conséquent ses points d'intersection avec $C(\lambda)$ bougent peu, ce qui signifie que beaucoup de courbure se concentre au voisinage de ces points. Rappelons que le vocable branche désigne un germe analytiquement irréductible de courbe, et en particulier une composante irréductible d'un germe de courbe.

Si l'on souhaite représenter au moyen d'un logiciel graphique une projection générique dans $\mathbf{R}^{3}$ d'une fibre de Milnor de courbe plane complexe, il sera impossible de le faire effectivement en respectant la courbure dès qu'il y aura plus d'une paire de Puiseux, en raison de ce caractère multi-échelles qui implique qu'en certains points la courbure paraîtra infiniment grande (ou petite) par rapport à celle d'autres points. Connaître a priori les échelles en jeu devrait permettre de corriger la représentation de telle manière qu'elle devienne utile.

Remerciements: Ce travail a été en partie déclenché par des questions de Langevin au second auteur, et Langevin lui-même a obtenu des résultats préliminaires, poursuivis dans une direction différente de celle suivie ici, la courbure des feuilletages, par P. Rouillé [Ro]. Nous voulons remercier Patrick Popescu-Pampu, élève à l'ENS, pour sa lecture aigüe et ses remarques pertinentes sur une version préliminaire.

\section{Invariants du type topologique}

Soit $f(x, y)=0, f(x, y) \in \mathbf{C}\{x, y\}$ et $f(0, y) \not \equiv 0$, une équation d'un germe irréductible de courbe plane à singularité isolée à l'origine $(C, 0) \subset\left(\mathbf{C}^{2}, 0\right)$ de multiplicité $n$. Si $x=0$ est transverse à $C$ il existe une paramétrisation de $C$ de la forme suivante:

$$
\left\{\begin{array}{l}
x=t^{n} \\
y=\sum_{i \geq n} a_{i} t^{i},
\end{array}\right.
$$

ce qui équivaut à la paramétrisation à la Newton-Puiseux $y=\sum_{i \geq n} a_{i} x^{i / n}$. Toutes les autres paramétrisations à la Newton-Puiseux de $C$ sont déduites de celle-ci par l'action du groupe $\mu_{n}$ des racines $n$-ième de l'unité determinée par $x^{1 / n} \rightarrow \omega x^{1 / n}$ où $\omega \in \mu_{n}$ est une racine primitive $n$-ième de l'unité. 
Soient $\left\{\beta_{0}, \ldots, \beta_{g}\right\} \subset \mathbf{N}$ les exposants caractéristiques de $C$ qui sont définis par récurrence de la manière suivante: $\beta_{0}=m(C)=n$, $\beta_{q+1}=\min \left\{i \in \mathbf{N}: a_{i} \neq 0\right.$ et $i \not \equiv 0\left(\bmod\right.$ p.g.c.d. $\left.\left.\left(\beta_{0}, \ldots, \beta_{q}\right)\right)\right\}$.

Il existe $g \geq 1$ minimal tel que p.g.c.d. $\left(\beta_{0}, \ldots, \beta_{g}\right)=1$.

Soient alors: $l_{0}=m(C)$ et $l_{q}=$ p.g.c.d. $\left(\beta_{0}, \ldots, \beta_{q}\right)=$ p.g.c.d. $\left(l_{q-1}, \beta_{q}\right)$. Il existe des paires d'entiers positifs $\left\{\left(m_{k}, n_{k}\right)_{k=1}^{g}\right\}$ tels que $\beta_{q}=m_{q} l_{q}, l_{q-1}=n_{q} l_{q}$ et p.g.c.d. $\left(m_{q}, n_{q}\right)=1$. Les paires $\left\{\left(m_{k}, n_{k}\right)_{k=1}^{g}\right\}$ sont appelés paires caractéristiques de Puiseux de $C$.

Soit $\Gamma(C)=\{(C, D) / C$ n'est pas une composante de $D\} \subset \mathbf{N}$ le semi-groupe de valeurs de $C$, où $(C, D)$ est la multiplicité d'intersection à l'origine de $C$ avec une courbe quelconque $D$.

D'après Zariski [Za1] il existe un système minimal de génerateurs $\left\{\bar{\beta}_{0}, \ldots \bar{\beta}_{g}\right\}$ du semi-groupe $\Gamma(C)$ qui vérifie $\bar{\beta}_{0}=\beta_{0}=m(C), \bar{\beta}_{1}=\beta_{1}$, et $\bar{\beta}_{q}=n_{q-1} \bar{\beta}_{q-1}+$ $\beta_{q}-\beta_{q-1}$ pour tout $q \in\{2, \ldots, g\}$. On voit ainsi que la donnée du semi-groupe $\Gamma(C)$ équivaut à celle des paires caractéristiques de Puiseux.

De plus si $C$ et $D$ sont deux branches à l'origine on appele contact de $C$ avec $D$ le nombre rationnel

$$
\operatorname{cont}(C, D)=n \max _{1 \leq i \leq n, 1 \leq j \leq m}\left\{\operatorname{ord}_{x}\left(y_{i}\left(x^{1 / n}\right)-z_{j}\left(x^{1 / m}\right)\right)\right\}
$$

où $m(C)=n, \quad m(D)=m, \quad\left\{y_{i}\left(x^{(1 / n)}\right)\right\}_{i=1}^{n}$ est l'ensemble des paramétrisations de $C$ et $\left\{z_{i}\left(x^{(1 / m)}\right)\right\}_{i=1}^{m}$ est l'ensemble des paramétrisations de $D$. Le contact entre $C$ et $D$ donne donc une mesure de la cö̈ncidence des paramétrisations à la Newton-Puiseux de $C$ et de $D$.

On doit remarquer aussi que

$$
\frac{\operatorname{cont}(C, D)}{m(C)}=\frac{\operatorname{cont}(D, C)}{m(D)} .
$$

Soient $(C, 0) \subset\left(\mathbf{C}^{2}, 0\right)$ un germe réduit de courbe analytique plane à singularité isolée à l'origine de multiplicité $n$, et $C=C_{1} \cup \cdots \cup C_{r}$ sa décomposition en composantes irréductibles. Notons $I$ l'ensemble $\{1, \ldots, r\}$ et $\left\{n_{i}=\beta_{0}^{i}, \ldots, \beta_{g_{i}}^{i}\right\}$ les exposants caractéristiques de $C_{i}, l_{k}^{i}=$ p.g.c.d. $\left(\beta_{0}^{i}, \ldots, \beta_{k}^{i}\right)$ où $k \in\left\{0, \ldots, g_{i}\right\}$, $\left\{\left(m_{k}^{i}, n_{k}^{i}\right)\right\}_{k=1}^{g_{i}}$ les paires caractéristiques de $C_{i}, \quad \Gamma\left(C_{i}\right)=<\left\{\overline{\beta_{0}^{i}}, \ldots, \overline{\beta_{g_{i}}^{i}}\right\}>$ le semi-groupe de valeurs de $C_{i}$ et $\alpha_{i j}=\operatorname{cont}\left(C_{i}, C_{j}\right)$ avec $j \neq i$.

Nous pouvons représenter la topologie de la courbe $C$ au moyen du diagramme d'Eggers (voir [E] et [GB1]). Rappellons sa définition.

A chaque branche $C_{i}$ de $C$ on associe un ensemble $S_{i}:=S_{i}^{1} \cup S_{i}^{2}$ où $S_{i}^{1}=$ $\left\{\frac{\beta_{k}^{i}}{n_{i}}\right\}_{k=1}^{g_{i}}$ et $S_{i}^{2}=\left\{\frac{\alpha_{i j}}{n_{i}}\right\}_{j \neq i}$.

On appelle chaîne élémentaire de $C_{i}$ le graphe $K_{i}$ défini comme suit:

1. Les sommets sont des points noirs et un point blanc; les points noirs sont en correspondance bijective avec les éléments de $S_{i}$ par une application $v$ que nous appelons valuation. 
2. Le sommet blanc n'a pas de valuation.

3. Les sommets sont reliés de la façon suivante:

Le sommet blanc est relié au sommet noir de valuation la plus grande par une arête, qui est discontinue si la valuation est un élément de $S_{i}^{2}-S_{i}^{1}$. Si on prend un sommet noir, disons $Q$, dont la valuation n'est pas maximale, il est relié au sommet de valuation supérieure la plus proche par une arête, qui est discontinue si $v(Q)$ est dans $S_{i}^{2}-S_{i}^{1}$.

Si on prend deux branches différentes $C_{i}, C_{j}$ de $C$, on appelle graphe partiel $K_{i j}$ de $C_{i}$ et $C_{j}$, le plus petit sous-graphe connexe de $K_{i}$ qui contient les sommets $Q \in K_{i}$ avec $v(Q) \leq \frac{\alpha_{i j}}{n_{i}}$. Les graphes partiels $K_{i j}$ et $K_{j i}$ sont égaux.

Finalement on définit le diagramme d'Eggers $T(C)$ de $C$ comme le graphe obtenu en identifiant les graphes partiels $K_{i j}, K_{j i}$ dans la réunion disjointe des chaînes élémentaires $K_{1}, \ldots, K_{r}$.

On voit aussitôt que deux germes de courbes réduites sont équisinguliers si et seulement si ils ont le même diagramme d'Eggers.

Exemple 1.1. Soit la courbe $C \equiv f=f_{1} \cdot f_{2}=0$ où $f_{1}(x, y)=\left(y^{2}-x^{3}\right)^{2}-$ $4 y x^{6}-x^{9}$ et $f_{2}(x, y)=\left(y^{2}-x^{3}\right)^{2}-4 y x^{5}-x^{7}$.

Les développements de Puiseux de $f_{1}(x, y)=0$ sont

$$
\left\{\begin{array}{l}
y_{1}=x^{3 / 2}+x^{9 / 4} \\
y_{2}=-x^{3 / 2}+i x^{9 / 4} \\
y_{3}=x^{3 / 2}-x^{9 / 4} \\
y_{4}=-x^{3 / 2}-i x^{9 / 4}
\end{array}\right.
$$

et les développements de Puiseux de $f_{2}(x, y)=0$ sont

$$
\left\{\begin{array}{l}
y_{1}=x^{3 / 2}+x^{7 / 4} \\
y_{2}=-x^{3 / 2}+i x^{7 / 4} \\
y_{3}=x^{3 / 2}-x^{7 / 4} \\
y_{4}=-x^{3 / 2}-i x^{7 / 4}
\end{array}\right.
$$

Donc $S_{1}^{1} \cup S_{1}^{2}=\left\{\frac{3}{2}, \frac{9}{4}\right\} \cup\left\{\frac{7}{4}\right\}$ et $S_{2}^{1} \cup S_{2}^{2}=\left\{\frac{3}{2}, \frac{7}{4}\right\} \cup\left\{\frac{7}{4}\right\}$.

Le diagramme d'Eggers de $C$ est

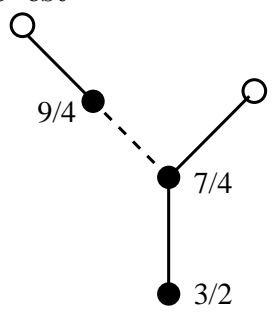


On appelle point base de $T(C)$ le sommet de $T(C)$ de valuation la plus petite.

A chaque sommet noir $Q$ de $T(C)$ on associe trois nombres :

$d_{1}(Q):=$ nombre d'arêtes discontinues de $T(C)$ qui sortent de $Q$ vers un sommet noir de valuation plus grande ou vers un sommet blanc de $T(C)$.

$d_{2}(Q):=$ nombre d'arêtes pleines de $T(C)$ qui sortent de $Q$ vers un sommet noir de valuation plus grande ou vers un sommet blanc de $T(C)$.

Nous dirons qu'un sommet est simple si $d_{1}(Q)+d_{2}(Q)=1$, un sommet $d e$ bifurcation sinon.

$k(Q):=$ nombre d'arêtes pleines entre $Q$ et le point base.

Soit $Q$ un sommet noir de $T(C)$ tel que $d_{2}(Q)>0$. Le fait que l'arête qui sort de $Q$ dans la chaîne élémentaire $K_{i}$ de $T(C)$ soit pleine équivaut à dire que la valuation de $Q$ est égale à $\frac{\beta_{k+1}^{i}}{n_{i}}$ où $k=k(Q)$.

Notons $I_{Q}$ l'ensemble $\left\{i \in I / Q \in K_{i}\right\}$ où $K_{i}$ est la chaine élémentaire de la branche $C_{i}$, et $I_{Q}^{*}$ le sous-ensemble de $I_{Q}$ vérifiant: $I_{Q}^{*}=I_{Q}$ si $d_{2}(Q)=0$ et

$I_{Q}^{*}=\left\{i \in I_{Q}: v(Q)=\frac{\beta_{k+1}^{i}}{n_{i}}\right\}$ si $d_{2}(Q)>0$.

\section{Calculs sur les branches de la courbe polaire}

Soient $f: \mathbf{C}^{2} \longrightarrow \mathbf{C}$ un morphisme défini dans un voisinage ouvert $V$ de l'origine et $l \equiv l(x, y): \mathbf{C}^{2} \longrightarrow \mathbf{C}$ une forme linéaire sur $\mathbf{C}^{2}$.

On appelle courbe de niveau $\lambda$ de $f$ la courbe $C(\lambda) \subset V$ d'équation $f(x, y)=\lambda$.

On appelle courbe polaire de $f$ dans la direction $l$ le lieu $C_{l}$ des points critiques dans $V$ de $(f, l): V \longrightarrow \mathbf{C}^{2}$.

Ainsi, si $P$ est un point de $C_{l}$ alors ou bien $P$ est un point singulier de $C(\lambda)$ ou bien $P$ est un point lisse de $C(\lambda)$ et la droite tangente à $C(\lambda)$ au point $P$ est parallèle à $l(x, y)=0$.

Remarque 2.1. La courbe polaire est vide dans un voisinage assez petit de $0 \mathrm{si}$ et seulement si $C \equiv f(x, y)=0$ est lisse en 0 . Nous nous plaçons dans le cas où $C$ est singulière.

Nous pouvons écrire l'équation d'une courbe polaire de $f$ sous la forme:

$$
\sigma \frac{\partial f}{\partial y}+\tau \frac{\partial f}{\partial x}=0, \quad(\sigma: \tau) \in \mathbf{P}^{1}(\mathbf{C}) .
$$

D'après les résultats généraux sur l'équisingularité (voir [Za3] et [Te1]), il existe un ouvert de Zariski $U$ de l'espace $\mathbf{P}^{1}(\mathbf{C})$ des directions de projection tel que pour $(\sigma: \tau) \in U$ les courbes polaires soient toutes équisingulières. Pour simplifier nous nous restreindrons à considérer l'intersection de $U$ avec l'ouvert affine $\sigma \neq 0$, 
poserons $\sigma=1$ et, pour $\tau \in U \cap \mathbf{A}^{1}$, noterons $P(\tau)$ la courbe polaire correspondante, appelée par abus de langage courbe polaire générique. C'est la fibre au dessus de $\tau \in \mathbf{A}^{1}$ de la surface $P$ définie dans un ouvert de $\mathbf{C}^{3}$ par

$$
\frac{\partial f}{\partial y}+\tau \frac{\partial f}{\partial x}=0
$$

Dans toute la suite nous supposerons que le choix de la coordonnée $x$ est assez général pour que le point $\tau=0$ appartienne à l'ouvert $U$.

Toujours d'après les résultats généraux sur l'équisingularité (voir loc.cit.), il existe un recouvrement de $U$ par des ouverts relativement compacts $\left(U_{\delta}\right)_{\delta}$ et pour chaque $\delta$ il existe un rayon $\epsilon(\delta)>0$ tel que dans l'ouvert $U_{\delta} \times \mathbf{B}_{\epsilon(\delta)}$, où $\mathbf{B}_{\epsilon}=\mathbf{B}(0, \epsilon)$ est la boule ce centre 0 et de rayon $\epsilon$, la famille des courbes polaires est une famille équisingulière de courbes planes et en particulier pour tout $\tau \in U_{\delta}$ le nombre des composantes irréductibles de $P(\tau)$ est constant et la surface $P$ de $U_{\delta} \times \mathbf{B}_{\epsilon(\delta)}$ réunion des $(P(\tau))_{\tau \in U_{\delta}}$ est réunion des surfaces $P_{q}$ paramétrées de la forme:

$$
P_{q} \cap\left(U_{\delta} \times \mathbf{B}_{\epsilon(\delta)}\right)=\left\{\begin{array}{l}
x=t_{q}^{m_{q}} \\
y=y\left(t_{q}, \tau\right) \quad \text { avec } y\left(t_{q}, \tau\right) \in \mathbf{C}\left\{t_{q}, \tau\right\}
\end{array}\right.
$$

$P(\tau)$ designe donc aussi bien la courbe polaire correspondant à la direction $\tau$ que la fibre au dessus de $\tau \in U$ de la surface $P$. Puisque la famille des $P(\tau)$ est équisingulière pour $\tau \in U$, nous nous permettrons souvent de supprimer le $\tau$ pour des caractères numériques tels que multiplicités, nombres d'intersection, qui sont constants pour $\tau \in U$, et de même pour les composantes irréductibles de $P$ que nous allons introduire:

Rappelons le résultat suivant:

Théorème 2.1 ([GB2]). Dans $U_{\delta} \times \mathbf{B}_{\epsilon(\delta)}$ les composantes irréductibles de $P(\tau)$ se rassemblent en $l$ ensembles (que nous appellerons "paquets") $\Gamma^{Q_{j}}(\tau)$ indexés par les sommets noirs $\left(Q_{j}\right)_{1 \leq j \leq l}$ de $T(C)$. De plus:

1. Toutes les composantes irréductibles d'un paquet $\Gamma^{Q_{j}}(\tau)$ ont le même contact avec chacune des branches de $C$ et cette condition de contact est donnée des deux manières équivalentes suivantes:

(a) Pour chaque composante irréductible $P_{q}(\tau)$ de $\Gamma^{Q_{j}}(\tau)$ et pour toute composante irréductible $C_{i}$ de $C$ telle que $Q_{j}$ appartient a la chaîne élémentaire de $C_{i}$ on a l'égalité:

$$
\frac{\left(C_{i}, P_{q}(\tau)\right)}{m\left(P_{q}(\tau)\right)}=\frac{n_{k}^{i} \overline{\beta_{k}^{i}}+n_{i} v\left(Q_{j}\right)-\beta_{k}^{i}}{n_{1}^{i} \ldots n_{k}^{i}}
$$

où $k:=k\left(Q_{j}\right)$.

(b) Chaque composante irréductible $P_{q}(\tau)$ de $\Gamma^{Q_{j}}(\tau)$ a un développement de 
Puiseux de la forme:

$$
\left\{\begin{array}{l}
x=t^{m_{q}} \\
y=a_{n_{i_{0}}} t^{m_{q}}+\cdots+a_{s n_{i_{0}}} t^{s m_{q}}+a_{\beta_{1}^{i_{0}}} t^{\tilde{\beta}_{1}^{i_{0}}}+\cdots+a_{\beta_{q}^{i_{0}}} t^{\tilde{\beta}_{q}^{i_{0}}}+\cdots+ \\
\quad+a_{n_{i_{0}} v\left(Q_{j}\right)-1} t^{\frac{m_{q}\left(n_{i_{0}} v\left(Q_{j}\right)-1\right)}{n_{i_{0}}}}+\sum_{s \geq m_{q} v\left(Q_{j}\right)} b_{s}(\tau) t^{s}
\end{array}\right.
$$

où $t$ est un paramètre uniformisant, l'indice $i_{0}$ est tel que $Q_{j}$ appartient à la chaîne élémentaire de la branche $C_{i_{0}}$ de $C$, l'entier $m_{q}:=m\left(P_{q}(\tau)\right)$ est la multiplicité à l'origine de $P_{q}(\tau)$, et les $b_{s}(\tau)$ sont dans $\mathbf{C}\{\tau\}$.

Ici le tilde indique que tous les exposants ont été divisés par un facteur commun $\frac{n_{i_{0}}}{m_{q}}$.

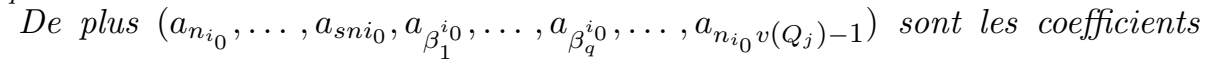
d'un développement de Puiseux de $C_{i_{0}}$, jusqu'au terme $n_{i_{0}} v\left(Q_{j}\right)-1$, et sont donc indépendants de $\tau$ pour toute valeur $\tau$ qui appartient à l'ouvert $U_{\delta}$ d'équisingularité. Ceci signifie qu'à division près des exposants par un facteur commun, le développement de Puiseux de $P_{q}(\tau)$ coïncide avec le développement de Puiseux de la branche $C_{i_{0}}$ de la courbe $C$ au moins jusqu'au dernier terme précédant la valeur $n_{i_{0}} v\left(Q_{j}\right)-1$. On ne peut être plus précis en général puisque le type topologique de la courbe polaire (et en particulier le nombre de ses composantes irréductibles) n'est pas déterminé par celui de la courbe $C$.

2. La multiplicité de $\Gamma^{Q_{j}}(\tau)$ est égale à $n_{1}^{i} \cdots n_{k}^{i}\left(d_{1}\left(Q_{j}\right)+n_{k+1}^{i} d_{2}\left(Q_{j}\right)-1\right)$ où l'index $i \in I_{Q_{j}}^{*}$.

L'égalité 2 est due a Eggers [E] et le résultat (a) est dû à Smith [S] et Merle [Me] dans le cas irréductible.

Remarque 2.2. La décomposition de $P(\tau)=\bigcup_{Q_{j}} \Gamma^{Q_{j}}(\tau)$ donne une décomposition dans $U_{\delta} \times \mathbf{B}_{\epsilon(\delta)}$ de la surface $P$ de la forme suivante: $P=\bigcup_{Q_{j}} \Gamma^{Q_{j}}$ avec $\Gamma^{Q_{j}}=$ $\bigcup_{\tau \in U_{\delta}} \Gamma^{Q_{j}}(\tau)$

Le développement (b) est une paramétrisation analytique de la surface $P_{q} \subset$ $U_{\delta} \times \mathbf{B}_{\epsilon(\delta)}$.

Du point de vue de (b) on peut montrer le résultat suplémentaire suivant qui généralise un résultat connu dans le cas irréductible [S], [T4]:

Lemme 2.1 ([GB2]). Si $Q$ est un sommet noir simple de $T(C)$ et $P_{q}(\tau)$ est une 
branche du paquet $\Gamma^{Q}(\tau)$ alors dans le développement de $y(t)$ de la branche $\Gamma(\tau)$ le terme $t^{m_{q} v(Q)}$ n'apparaît pas.

Nous allons utiliser le théorème pour calculer en fonction de $\lambda$ et $\tau$ les coordonnées des points d'intersection de la courbe de niveau $C(\lambda)$ et la courbe polaire $P(\tau)$.

Pour simplifier la notation dans le calcul qui suit nous noterons $P_{q}$ une des composantes irréductibles de la surface $\Gamma^{Q_{j}}$, que nous continuerons par abus de langage d'appeler "branche". Nous noterons $m_{q}$ la multiplicité à l'origine de $P_{q}(\tau)$ et $e_{q}+m_{q}$ son nombre d'intersection en 0 avec $C$, pour $\tau$ générique (voir [T3]).

Nous noterons $\gamma_{q}$ le plus petit exposant plus grand ou égal à $m_{q} v\left(Q_{j}\right)$ apparaissant dans le développement de Puiseux $y_{q}(t, \tau)$ de $P_{q}(\tau)$ en puissances de $t$. Comme l'a montré E. Casas dans [Ca2] dans le cas irréductible, ce nombre ne dépend pas seulement du paquet $\Gamma^{Q_{j}}$, mais bien de la composante $P_{q}$ choisie. E. Casas a calculé (Prop. 1.1 de [Ca2]) la valeur de $\gamma_{q}$ pour une courbe générique dans sa classe d'équisingularité.

On peut donc écrire la partie (b) du théorème pour la surface $P_{q}$ sous la forme suivante:

$$
P_{q} \equiv\left\{\begin{array}{l}
x=t^{m_{q}} \\
y_{q}(t, \tau)=a_{n_{i}} t^{m_{q}}+\cdots+a_{s n_{i}} t^{s m_{q}}+\cdots+b_{\gamma_{q}}(\tau) t^{\gamma_{q}}+\cdots
\end{array}\right.
$$

où les coefficients des puissances de $t$ plus petites que $\gamma_{q}$ sont indépendants de $\tau$ car ils coïncident avec les coefficients d'une développement de Puiseux d'une branche $C_{i}$ de $C$ (voir [GB2]).

Nous allons utiliser aussi le fait suivant: Pour tout $\tau \in U_{\delta}$, la multiplicité d'intersection de $P_{q}(\tau)$ avec $C$ est strictement plus grande que $m_{q}$ car $C$ est singulière. Cela permet d'écrire $\left(C, P_{q}(\tau)\right)=e_{q}+m_{q}$ avec $e_{q}>0$. En fait, la somme sur toutes les composantes $P_{q}(\tau)$ de $P(\tau)$ des $e_{q}$ est égale à $\mu^{(2)}(C)$ (voir [Te3]). Ainsi l'égalité $\left(C, P_{q}(\tau)\right)=e_{q}+m_{q}$ signifie que l'on a une identité

$$
f\left(t^{m}, y_{q}(t, \tau)\right)=c_{e_{q}+m_{q}}(\tau) t^{e_{q}+m_{q}}+\cdots \quad \text { avec } c_{e_{q}+m_{q}}(\tau) \neq 0
$$

Lemme 2.2. Le coefficient de $t^{k}$ dans la série $f\left(t^{m_{q}}, y_{q}(t, \tau)\right) \in \mathbf{C}\{t, \tau\}$ est indépendant de $\tau$ pour $k$ plus petit que $e_{q}+\gamma_{q}$.

\section{Démonstration}

Il faut calculer l'ordre en $t$ de $\frac{\partial f\left(t^{m_{q}}, y_{q}(t, \tau)\right)}{\partial \tau}$.

Mais $\frac{\partial f\left(t^{m_{q}}, y_{q}(t, \tau)\right)}{\partial \tau}=\left(\frac{\partial f}{\partial x}\right)_{P_{q}}\left(\frac{\partial x}{\partial \tau}\right)_{P_{q}}+\left(\frac{\partial f}{\partial y}\right)_{P_{q}}\left(\frac{\partial y}{\partial \tau}\right)_{P_{q}}$, et comme 


$$
\begin{aligned}
&\left(\frac{\partial x}{\partial \tau}\right)_{P_{q}}=0, \text { on a } \\
& \qquad \frac{\partial f\left(t^{m_{q}}, y_{q}(t, \tau)\right)}{\partial \tau}=\left(\frac{\partial f}{\partial y}\right)_{P_{q}}\left(\frac{\partial y}{\partial \tau}\right)_{P_{q}} .
\end{aligned}
$$

Puisque dans le développement de $y_{q}(t, \tau)$ les puissances de $t$ de degré plus petit que $\gamma_{q}$ sont indépendantes de $\tau$ on a:

$$
\operatorname{ord}_{t}\left(\frac{\partial y}{\partial \tau}\right)_{P_{q}} \geq \gamma_{q}
$$

De plus $\operatorname{ord}_{t}\left(\frac{\partial f\left(t^{m_{q}}, y_{q}(t, \tau)\right)}{\partial y}\right)=e_{q}$, il suffit de se placer dans des coordonnées $(x, y)$ telles que $\tau=0$, donc $P \equiv \frac{\partial f}{\partial y}=0$ et $\frac{\partial f\left(x_{q}(t), y_{q}(t)\right)}{\partial t}=\frac{\partial f}{\partial x} \cdot \frac{\partial x}{\partial t}$, alors

$$
\operatorname{ord}_{t}\left(\frac{\partial f\left(x_{q}(t), y_{q}(t)\right)}{\partial t}\right)=e_{q}+m_{q}-1
$$

et comme $\operatorname{ord}_{t}\left(\frac{\partial x}{\partial t}\right)=m_{q}-1$ on a $\operatorname{ord}_{t}\left(\frac{\partial f}{\partial x}\right)=e_{q}$.

(Pour plus de détails voir le Lemme 3.3.2 de [GB1]).

Alors $\operatorname{ord}_{t}\left(\frac{\partial f\left(t^{m_{q}}, y_{q}(t, \tau)\right)}{\partial \tau}\right) \geq e_{q}+\gamma_{q}$.

Il en résulte que nous pouvons écrire

$$
f\left(t^{m_{q}}, y_{q}(t, \tau)\right)=\sum_{\alpha=e_{q}+m_{q}}^{e_{q}+\gamma_{q}-1} c_{\alpha} t^{\alpha}+\sum_{\alpha \geq e_{q}+\gamma_{q}} c_{\alpha}(\tau) t^{\alpha}
$$

Il nous reste à en tirer des expressions pour le développement de $x$ et $y$ en fonction de $\tau$, pour chaque valeur de $\lambda$. Or nous avons les faits suivants:

1. D'une part la surface de $\mathbf{C}^{4}$ ayant pour équations

$$
f(x, y)=\lambda, \quad \frac{\partial f}{\partial y}+\tau \frac{\partial f}{\partial x}=0
$$

admet localement sur $U_{\delta}$ et pour $x, y$ et $\lambda$ assez petits une représentation paramétrique sous forme d'un développement de $t$ en puissances de $\lambda^{\frac{1}{e_{q}+\gamma_{q}}}$, à coefficients analytiques en $\tau$. En effet, en utilisant la paramétrisation simultanée que nous avons vue plus haut de chacune des composantes irréductibles 
de la surface $S$ d'équation $\frac{\partial f}{\partial y}+\tau \frac{\partial f}{\partial x}=0$, et en la substituant dans $f(x, y)-\lambda$, il vient

$$
t^{e_{q}+m_{q}} V(t, \tau)-\lambda=0
$$

où $V(t, \tau) \in \mathbf{C}\{t, \tau\}$ est une unité.

Si maintenant on considére la surface $S^{\prime} \subset \mathbf{C}^{3}$ définie par cette équation, le discriminant de sa projection dans le plan des $(\tau, \lambda)$ est défini ensemblistement par $\lambda=0$ et d'après le critère discriminant de Zariski (voir [Za3]) cela implique l'existence d'un développement de $t$ en puissances de $\lambda^{\frac{1}{e_{q}+m_{q}}}$, à coefficients holomorphes en $\tau$.

2. D'autre part un calcul sans difficulté (voir page 214 de [GB1]) donne, pour $\lambda$ fixé, l'égalité

$$
\left(\frac{\partial t}{\partial \tau}\right)_{P_{q}}=-\frac{\left(\frac{\partial f}{\partial y}\right)_{P_{q}}\left(\frac{\partial y}{\partial \tau}\right)_{P_{q}}}{\left(\frac{\partial f}{\partial x}\right)_{P_{q}}\left(\frac{\partial x}{\partial t}\right)_{P_{q}}+\left(\frac{\partial f}{\partial y}\right)_{P_{q}}\left(\frac{\partial y}{\partial t}\right)_{P_{q}}} .
$$

On en déduit que l'ordre en $t$ de $\left(\frac{\partial t}{\partial \tau}\right)_{P_{q}}$ est au moins $\gamma_{q}-m_{q}+1$. Par inversion de l'équation $f\left(t^{m_{q}}, y_{q}(t, \tau)\right)=\lambda$ comme ci-dessus on obtient un développement de Puiseux de $t$ en fonction de $\lambda$. Puisque $t$ est de l'ordre de $\lambda^{\frac{1}{e_{q}+m_{q}}}$ on déduit de la remarque ci-dessus que l'ordre en $\lambda$ de $\left(\frac{\partial t}{\partial \tau}\right)_{P_{q}}$ est au moins $\frac{\gamma_{q}-m_{q}+1}{e_{q}+m_{q}}$. Le développement de $t$ en fonction de $\lambda$ s'écrit donc

$$
t=\sum_{\alpha=1}^{\gamma_{q}-m_{q}} d_{\alpha} \lambda^{\frac{\alpha}{e_{q}+m_{q}}}+\sum_{\alpha \geq \gamma_{q}-m_{q}+1} d_{\alpha}(\tau) \lambda^{\frac{\alpha}{e_{q}+m_{q}}}
$$

avec $d_{\alpha}(\tau) \in \mathbf{C}\{\tau\}$ et $d_{1} \neq 0$, où l'écriture signifie ici et dans la suite, que la plus petite puissance de $\lambda$ dont le coefficient peut dépendre de $\tau$ est $\lambda^{\frac{\gamma_{q}-m_{q}+1}{e_{q}+m_{q}}}$.

Finalement, en utilisant ce que nous venons de voir pour calculer $\frac{\partial x}{\partial \tau}$ et $\frac{\partial y}{\partial \tau}$, on voit que l'on a des développements de Puiseux pour les coordonnées $x$ et $y$ des points de $P_{q}(\tau) \cap C(\lambda)$ dans l'espace $(x, y, \lambda, \tau)$ en fonction de $\lambda$ et $\tau$ de la forme suivante:

$$
\left\{\begin{array}{l}
x=\sum_{\alpha=m_{q}}^{\gamma_{q}-1} g_{\alpha} \lambda^{\frac{\alpha}{e_{q}+m_{q}}}+\sum_{\alpha \geq \gamma_{q}} g_{\alpha}(\tau) \lambda^{\frac{\alpha}{e_{q}+\gamma_{q}}} \quad \text { où } g_{m_{q}} \neq 0 \\
y=\sum_{\alpha=m_{q}}^{\gamma_{q}-1} h_{\alpha} \lambda^{\frac{\alpha}{e_{q}+m_{q}}}+\sum_{\alpha \geq \gamma_{q}} h_{\alpha}(\tau) \lambda^{\frac{\alpha}{e_{q}+m_{q}}}
\end{array}\right.
$$


Ainsi quand $\tau$ varie dans $U_{\delta}$, les coordonnées des points de $P(\tau)$ où la tangente à la courbe de niveau $f(x, y)=\lambda$ a pour direction $\tau$ ne dépendent effectivement de $\tau$ qu'à partir de l'exposant $\gamma_{q}$.

Le calcul précédent a été fait dans un ouvert $U_{\delta} \times \mathbf{B}_{\epsilon(\delta)}$, mais il est clair que la forme du résultat est localement constante sur $U$, et donc indépendante de l'ouvert $U_{\delta}$ choisi.

\section{Concentration de courbure}

Le but de cette section est de montrer que la courbure des courbes de niveau $C(\lambda) \equiv f(x, y)=\lambda$ de $C$ est concentrée autour des points d'intersection de ces fibres avec les branches des courbes polaires génériques de $C$.

\subsection{Les boules}

Soit $\xi_{q, 0}^{Q}(\lambda)$ le point

$$
\xi_{q, 0}^{Q}(\lambda):=\left(\sum_{\alpha=m_{q}}^{\gamma_{q}-1} g_{\alpha} \lambda^{\frac{\alpha}{e_{q}+m_{q}}}, \sum_{\alpha=m_{q}}^{\gamma_{q}-1} h_{\alpha} \lambda^{\frac{\alpha}{e_{q}+m_{q}}}\right)
$$

dont les coordonnées sont les parties indépendants de $\tau$ des développements (5). S'il n'existe pas de coefficients indépendants de $\tau$ dans l'expression (5) alors $\xi_{q, 0}^{Q}(\lambda):=(0,0)$.

Notons $\xi_{q, l}^{Q}(\lambda)$ le point obtenu en remplaçant dans $\xi_{q, 0}^{Q}(\lambda)$ le nombre complexe $\lambda$ par $\omega^{l} \lambda$ où $\omega$ est une racine primitive $\left(e_{q}+m_{q}\right)$ - ième de l'unité et $l \in\left\{0,1, \ldots,\left(e_{q}+m_{q}-1\right)\right\}$.

Proposition 3.1. Soit $T \subset U$ un compact quelconque. Alors il existe $c(T) \in \mathbf{R}^{+}$ et $\varepsilon_{0}>0$ tels que pour tout $\tau \in T$ et pour tout $\epsilon<\epsilon_{0}$ les points d'intersection de $C(\lambda)$ avec la branche $P_{q}(\tau)$ du paquet $\Gamma^{Q}$ de $P(\tau)$ sont dans les boules fermées de centre $\xi_{q, l}^{Q}(\lambda)$ et de rayon $c(T)|\lambda|^{\frac{\gamma_{q}}{e_{q}+m_{q}}}$ quand $\lambda$ tend vers 0 .

\section{Démonstration}

$T$ est recouvert par des ouverts $U_{\delta} \cap T$. Soit $(x, y)$ un point de $C(\lambda) \cap P_{q}$. Il existe 
$l \in\left\{0, \ldots,\left(e_{q}+m_{q}-1\right)\right\}$ tel que

$$
\begin{aligned}
M & =\left\|(x, y)-\xi_{q, l}^{Q}(\lambda)\right\| \\
& =\|\lambda^{\frac{\gamma_{q}}{e^{q}+m_{q}}} \underbrace{\left(\sum_{\alpha \geq \gamma_{q}} g_{\alpha}(\tau) \lambda^{\frac{\alpha-\gamma_{q}}{e_{q}+m_{q}}}, \sum_{\alpha \geq \gamma_{q}} h_{\alpha}(\tau) \lambda^{\frac{\alpha-\gamma_{q}}{e_{q}+m_{q}}}\right)}_{\rho}\| \\
& =|\lambda|^{\frac{\gamma_{q}}{e_{q}+m_{q}}}\|\rho\| .
\end{aligned}
$$

Mais les $g_{s}(\tau), h_{s}(\tau)$ sont holomorphes et restent donc bornées sur le compact $T$. Ainsi quand $\lambda$ assez petit il existe une constante $c(T) \in \mathbf{R}^{+}$et il existe $\sigma(T) \in \mathbf{R}^{+}$ tels que pour $\tau \in T$ et $\lambda$ plus petit que $\sigma(T)$ on ait $\|\rho\| \leq c(T)$ et donc

$$
M \leq c(T)|\lambda|^{\frac{\gamma_{q}}{e_{q}+m_{q}}} .
$$

Plus précisément:

Proposition 3.2. Soit $T \subset U$ un compact quelconque. Soient $\Gamma^{Q}$ un paquet de la surface $P$ avec $v(Q)>1$ et $P_{q}$ une surface irréductible de ce paquet. Pour tout $\tau \in T$ et pour tout $|\lambda|$ assez petit les points d'intersection dans $\mathbf{C}^{2}$ de $P_{q}(\tau)$ et $C(\lambda)$ sont dans les boules $\mathbf{B}\left(\xi_{q, l}^{Q}(\lambda),|\lambda|^{\rho(Q)}\right)$ où $l \in\left\{0, \ldots,\left(e_{q}+m_{q}-1\right)\right\}$ et

$$
\rho(Q)=\left\{\begin{array}{l}
\frac{v(Q)}{\epsilon(Q)} \text { si } Q \text { est simple } \\
\frac{v(\tilde{Q})}{\epsilon(Q)} \text { si } Q \text { est un point de bifurcation différent du point base } \\
\frac{1}{\epsilon(Q)} \text { si } Q \text { est le point base et est un sommet de bifurcation }
\end{array}\right.
$$

où $\epsilon(Q)$ est l'invariant polaire associé au paquet $\Gamma^{Q}$ pour toute branche $P_{q}(\tau)$ de $\Gamma^{Q}$ et $\tilde{Q}$ est le prédécesseur de $Q$ sur la chaîne joignant le point base à $Q$.

Remarque 3.1. Rappellons que l'invariant polaire associé au paquet $\Gamma^{Q}$, introduit dans $(c f[\mathrm{Te} 3])$, où il est noté $\frac{e_{q}+m_{q}}{m_{q}}$ pour chaque branche $P_{q}$, de multiplicité $m_{q}$ du paquet $\Gamma^{Q}$, est $\frac{\left(C, P_{q}(\tau)\right)}{m\left(P_{q}(\tau)\right)}$ pour toute branche $P_{q}(\tau)$ de $\Gamma^{Q}(\tau)$ et est un invariant de type topologique de la courbe $C$ (voir [Te3], [GB2]). 
Remarque 3.2. S'il existe un sommet de valuation 1 dans $T(C)$ ce ne peut être que le point base. Cela signifie d'après le lemme 3.1 de [GB2] que la courbe $C$ contient au moins deux branches transverses. Dans ce cas-là nous noterons $\Gamma^{(0)}(\tau)$ le paquet de branches de la courbe polaire correspondant au point base.

\section{Démonstration}

Si $Q$ est un sommet simple, d'après le lemme 2.1 , on a $\gamma_{q}>m_{q} v(Q)$, donc il existe $\eta \in \mathbf{N}-\{0\}$ tel que $\eta=\gamma_{q}-m_{q} v(Q)$.

Ainsi d'après la proposition 3.1 si $(x, y) \in C(\lambda) \cap P_{q}$ il existe $l \in\left\{0, \ldots,\left(e_{q}+\right.\right.$ $\left.\left.m_{q}-1\right)\right\}$ et $c(T) \in \mathbf{R}^{+}$tels que

$$
M=\left\|(x, y)-\xi_{q, l}^{Q}(\lambda)\right\| \leq c(T)|\lambda|^{\frac{\gamma_{q}}{e_{q}+m_{q}}}=\underbrace{c(T)|\lambda|^{\frac{\eta}{e_{q}+m_{q}}}}_{\sigma}|\lambda|^{\frac{m_{q} v(Q)}{e_{q}+m_{q}}}
$$

et pour $|\lambda|$ assez petit on a $\sigma<1$, donc $M<|\lambda|^{\frac{v(Q)}{\epsilon(Q)}}$.

Soit maintenant $Q$ un sommet de bifurcation de $T(C)$ différent du point base de $T(C)$. Ainsi

$$
\gamma_{q} \geq m_{q} v(Q)>m_{q} v(\tilde{Q})
$$

où $\tilde{Q}$ est le prédécesseur de $Q$ sur la chaîne joignant le point-base à $Q$. Un raisonnement comme ci-dessus donne le résultat.

Cependant si $Q$ est le point base et est un point de bifurcation, alors $\gamma_{q} \geq$ $m_{q} v(Q)>m_{q}$ car $v(Q)>1$. On obtient le résultat en raisonnant comme cidessus.

Remarquons alors que pour $\lambda$ assez petit tout point d'intersection de $P(\tau)(C)-$ $\Gamma^{(0)}$ avec la courbe de niveau $C(\lambda)$ est dans une des boules que nous venons d'introduire dans la proposition ci-dessus.

Ainsi nous avons associé à chaque surface $P_{q}$ d'un paquet $\Gamma^{Q}$ de la surface $P$ des boules en nombre $e_{q}+m_{q}$ ayant toutes le même rayon pour $\lambda$ fixé. Si on prend une autre surface $P_{q^{\prime}}$ du paquet $\Gamma^{Q}$ les rayons des boules associées à $P_{q^{\prime}}$ et à $P_{q}$ coïncident pour $\lambda$ fixé. En conséquence, toutes les boules associées à des branches du même paquet de la polaire ont le même rayon pour $\lambda$ fixé.

Proposition 3.3. Il existe une famille finie $\left\{B_{q, l}^{Q}(\lambda):=B\left(\xi_{q, l}^{Q}(\lambda),|\lambda|^{\rho(Q)}\right)\right\}_{Q, q, l}$ de boules dont

1. Les centres et les rayons dépendent explicitement de $\lambda$.

2. Tout point d'intersection de $P(\tau)-\Gamma^{(0)}$ avec la fibre $C(\lambda)$ pour $\lambda$ assez petite est dans une de ces boules.

3. Les exposants $\rho(Q)$ qui apparaissent dans les rayons des boules ne dépendent que de la topologie de $C$.

\section{Démonstration}

1 et 2 résultent de ce que nous venons de voir, et 3 du fait que l'invariant po- 
laire $\epsilon(Q)$ et le diagramme d'Eggers sont des invariants topologiques (voir [Te2] et [GB2]).

Tandis que les rayons des boules de la proposition 3.2 dépendent seulement de la valeur de $\lambda$ et de la topologie de la courbe $C$, leurs centres dépendent en général du type analytique de $C$ et pas seulement du type topologique.

Etudions maintenant le cas où le point base de $T(C)$ est de valuation 1.

Lemme 3.1. Soit $\Gamma^{(0)}$ le paquet de la courbe polaire $P(\tau)$ dont le sommet associé $Q^{(0)}$ vérifie v $\left(Q^{(0)}\right)=1$. Alors

1. $\left(\Gamma^{(0)}, C\right)=(t-1) m(C)$ où $t$ est le nombre de composantes tangentielles de $C$. 2. $\Gamma^{(0)}$ est formé des branches de $P(\tau)$ qui sont transverses à $C$.

\section{Démonstration}

S'il n'existe aucun sommet de valuation égale à 1 alors $\Gamma^{(0)}$ est vide et $\left(\Gamma^{(0)}, C\right)=$ 0 . Cependant s'il existe un sommet $Q^{(0)}$ de valuation égale à 1 , alors $\left(\Gamma^{(0)}, C\right)=$ $\sum_{P_{q}(\tau) \in \Gamma^{(0)}}\left(P_{q}(\tau), C\right)$ où la somme est sur toutes les branches $P_{q}$ du paquet $\Gamma^{(0)}$. Comme la valuation de $Q^{(0)}$ est égale à $1, Q^{(0)}$ est le point base de $T(C)$ et $\operatorname{cont}\left(C_{i}, P_{q}\right)=n_{i}$ pour toute branche $C_{i}$ de $C$ et pour toute branche $P_{q}$ de $\Gamma^{(0)}$. Ainsi $\left(C_{i}, P_{q}\right)=n_{i} m_{q}$ et $\left(C, \Gamma^{(0)}\right)=m(C) m\left(\Gamma^{(0)}\right)$, c'est-à-dire que $\Gamma^{(0)}$ est transverse à $C$. Etant donné que chaque composante de la courbe polaire qui est transverse à $C$ est dans le paquet correspondant à $Q^{(0)}, \Gamma^{(0)}$ est la partie de $P(\tau)$ qui est transverse à $C$. De plus $m\left(\Gamma^{(0)}\right)=d_{1}\left(Q^{(0)}\right)-1=t-1$ et $\left(\Gamma^{(0)}, C\right)=(t-1) m(C)$.

Remarque 3.3. Ici et dans la suite, les $P_{q}$ sont des composantes du paquet $\Gamma^{Q}$; les indices $q$ et $Q$ ne sont donc pas indépendants.

\subsection{La courbure}

Rappellons qu'à une varieté réelle $V^{p} \subset \mathbf{R}^{\mathbf{N}}$ on peut associer en tout point $z \in V$ une courbure, dite de Lipschitz-Killing (voir $[\mathrm{F}]$ pour une définition précise) comme suit: A chaque vecteur normal unitaire $n \in \mathbf{S}^{N-p-1}$ à $V$ dans $\mathbf{R}^{N}$ en $z$ associons la projection orthogonale $P_{n}$ de $V$ dans l'espace vectoriel engendré par $T_{V, z}$ et $n$. Cette projection a pour image une hypersurface de $\mathbf{R}^{p+1}$ qui est non singulière en $z$, et on note $\gamma(V, n, z)$ sa courbure de Gauss au point $P_{n}(z)$. La courbure de Lipschitz-Killing est

$$
K(V, z)=c(N, p) \int_{\mathbf{S}^{N-p-1}} \gamma(V, n, z) d n
$$

où $c(N, p)$ est une constante de normalisation qui ne dépend que de $N$ et $p$. On a alors: 
Théorème 3.1 (formule d'échange [La]). Soit $W$ un ouvert d'une courbe holomorphe de $\mathbf{C}^{2}$; notons $K$ la courbure de Lipschitz-Killing de $W$ pour la métrique induite et $|\mu(W, L)|$ le nombre des points critiques de la projection de $W$ parallèlement à une direction de droite complexe $L \in \mathbf{P}^{1}(\mathbf{C})$. On a l'égalité ( où les intégrales sont prises par rapport aux mesures naturelles):

$$
\int_{W}|K| d v=2 \pi \int_{\mathbf{P}^{1}(\mathbf{C})}|\mu(W, L)| d L
$$

On déduit de ce théorème et de l'égalité $\mu^{(2)}+\mu^{(1)}=\mu^{(2)}(C)+\mu^{(1)}(C)=$ $(P(\tau), C)$ démontrée par Teissier (voir [Te1], [Te3]) le résultat suivant:

Théorème 3.2 (Langevin ([La])). On a l'égalité:

$$
\lim _{\epsilon, \lambda \rightarrow 0,|\lambda|<<\epsilon} \int_{C(\lambda)_{\epsilon}}|K| d v=2 \pi\left(\mu^{(2)}+\mu^{(1)}\right)
$$

où $\mu^{(2)}$ est le nombre de Milnor de $C$ et $\mu^{(1)}+1$ est la multiplicité $m(C)$ de $C$ en 0 .

Remarque 3.4. On peut vérifier en suivant la démonstration que l'écriture $\lim _{\epsilon, \lambda \rightarrow 0,|\lambda|<<\epsilon}$ peut être interprétée comme ceci: il existe dans le plan des $(\epsilon,|\lambda|)$ une courbe analytique réelle, ne dépendant que du choix des coordonnées et de l'équation de $C$, et admettant un développement de Puiseux

$$
|\lambda|=c_{k} \epsilon^{\frac{k}{p}}+c_{k+1} \epsilon^{\frac{k+1}{p}}+\cdots
$$

telle que la limite ait la valeur annoncée tant que $(\epsilon,|\lambda|)$ tendent vers 0 dans le premier quadrant en restant sous le graphe de la courbe. Cette condition sur le passage à la limite peut être interprétée ainsi dans la suite de cette article.

Le résultat de Langevin mesure la quantité totale de courbure de $C(\lambda)$ qui se concentre au point singulier 0 de $f(x, y)$ lorsque $\epsilon, \lambda \rightarrow 0$. Le résultat principal de cet article décrit de manière bien plus précise comment cette courbure se répartit asymptotiquement sur la fibre $C(\lambda)$ lorsque $(\epsilon, \lambda)$ tend vers $(0,0),|\lambda|<<\epsilon$.

Théorème 3.3. Reprenons les notations de la proposition (3.3). Soit $\Gamma^{(0)}$ le paquet de la courbe polaire $P(\tau)$ dont le sommet associé $Q^{(0)}$ vérifie $v\left(Q^{(0)}\right)=1$. Alors

$$
\lim _{\epsilon, \lambda \rightarrow 0,|\lambda|<<\epsilon}\left(\int_{C(\lambda)_{\epsilon}}|K| d v-\int_{C(\lambda)_{\epsilon} \cap\left(\bigcup_{Q, q, l} B_{q, l}^{Q}(\lambda)\right)}|K| d v\right)=2 \pi(t-1) m(C)
$$


où $t$ est le nombre de composantes tangentielles de $C$.

\section{Démonstration}

Prenons $W=C(\lambda)_{\epsilon}$. D'après la formule d'échange et la Proposition 3.3 on a l'inégalité

$$
\int_{C(\lambda)_{\epsilon} \cap\left(\bigcup_{Q, q, l} B_{q, l}^{Q}(\lambda)\right)}|K| d v \geq 2 \pi\left[(P(\tau), C)-\left(\Gamma^{(0)}, C\right)\right]
$$

Mais d'après le théorème (3.2), l'inégalité (9) et l'égalité $\mu^{(2)}+\mu^{(1)}=(C, P(\tau))$ on en déduit:

$$
\lim _{\lambda, \epsilon \rightarrow 0,|\lambda|<<\epsilon}\left(\int_{C(\lambda)_{\epsilon}}|K| d v-\int_{C(\lambda)_{\epsilon} \cap\left(\bigcup_{Q, q, l} B_{q, l}^{Q}(\lambda)\right)}|K| d v\right)=2 \pi\left(\Gamma^{(0)}, C\right)
$$

D'où le résultat d'après le lemme 3.1.

Corollaire 3.1. Etant donné un germe de morphisme analytique complexe $f:\left(\mathbf{C}^{2}, 0\right) \rightarrow(\mathbf{C}, 0)$ à fibre réduite, pour tout représentant assez petit, il existe un système de boules $B_{q, l}^{Q}(\lambda)$ dont les centres, décrits au début de la section 3.1, sont sur des courbes analytiques proches des composantes irréductibles d'une courbe polaire relative générique de $f$ et dont les rayons sont de la forme $|\lambda|^{\rho(Q)}$, où les $\rho(Q)$ sont des nombres rationnels ne dépendant que du type topologique du germe de courbe $(C, 0)=\left(f^{-1}(0), 0\right)$, décrits dans la Proposition 3.2. Ces boules ont la propriété que lorsque $\epsilon$ et $\lambda$ tendent vers $0,|\lambda|<<\epsilon$, une partie de la courbure de Lipschitz-Killing de la fibre de Milnor $C(\lambda)_{\epsilon}$ se concentre asymptotiquement dans la réunion de ces boules. La valeur de cette partie tend vers $2 \pi\left(\mu^{(2)}+(2-t) m-1\right)$ où $m$ est la multiplicité de $(C, 0)$ et $t$ le nombre de ses composantes tangentielles.

Nous dirons qu'il y a concentration de courbure sur $C(\lambda)$ si l'on peut montrer qu'une partie de courbure est asymptotiquement contenue dans l'intersection avec $C(\lambda)$ de boules dont le rayon tend vers 0 avec $|\lambda|$. Le terme $2 \pi\left(\Gamma^{(0)}, C\right)$ mesure la partie "diffuse" de la courbure. Les deux cas extrêmes sont:

1. $\left(\Gamma^{(0)}, C\right)=0$, c'est-à-dire que toutes les composantes de $C$ ont la même tangente (Lemme 3.1) et alors toute la courbure se concentre asymptotiquement dans des boules évanescentes. C'est en particulier le cas si $C$ est analytiquement irréductible en 0 .

2. $\left(\Gamma^{(0)}, C\right)=(P, C)$, c'est-à-dire que toutes les branches de la polaire sont transverses à $C$. Cela signifie que $\mu^{(2)}+\mu^{(1)}=\left(\mu^{(1)}+1\right) \mu^{(1)}$, donc $\mu^{(2)}=\left(\mu^{(1)}\right)^{2}$, et on sait ([Te3]) que cela implique que $C$ est réunion de $\mu^{(1)}+1$ branches lisses 2 à 2 transverses, donc $t=\mu^{(1)}+1$. 
Remarque 3.5. On doit remarquer que les boules où se concentre asymptotiquement la courbure de $C(\lambda)$ quand $\lambda \rightarrow 0$ sont indépendantes de $\tau$, que leurs rayons dépendent seulement de $\lambda$ et de la topologie de $C$, mais en principe leurs centres dépendent du type analytique de $C$. Bien que les centres dépendent du type analytique, on peut donc contrôler la "taille" et la géométrie de la région où se concentre asymptotiquement la courbure de $C(\lambda)$ quand $\lambda \rightarrow 0$ à partir de la topologie de la courbe $C$.

\subsection{Le cas d'une branche}

Soit $f(x, y)=0$ une équation pour un germe irréductible $(C, 0)$ de courbe plane décrite paramétriquement dans des coordonnées générales par le développement de Puiseux :

$$
\begin{aligned}
& x=t^{m} \\
& y=a_{m} t^{m}+\ldots+a_{k m} t^{k m}+a_{\beta_{1}} t^{\beta_{1}}+\ldots+a_{\beta_{j}} t^{\beta_{j}}+\ldots+a_{\beta_{g}} t^{\beta_{g}}+\ldots
\end{aligned}
$$

Reprenons les notations du $\S 1$. Dans le cas d'une branche, l'arbre d'Eggers consiste en une chaîne élémentaire contenant $g$ sommets noirs, que nous noterons $Q(j)$ par valuations croissantes, et pour chaque $Q(j)$, on a avec les notations ci-dessus

$$
\rho(Q(j))=\frac{\beta_{j}}{m} \frac{m_{q}}{e_{q}+m_{q}} \text { pour } q \in Q(j),
$$

la notation $q \in Q(j)$ indique que la composante $\Gamma_{q}$ de la courbe polaire appartient à ce paquet.

Proposition 3.4. Les $\rho_{j}=\rho(Q(j))$ forment une suite croissante.

En effet, d'après le Théorème de Smith et Merle ([S], [Me]), nous savons que $\frac{m_{q}}{e_{q}+m_{q}}$ est égal à $\frac{n_{1} \ldots n_{j-1}}{\overline{\beta_{j}}}$, ce qui donne après simplification $\rho_{j}=\frac{m_{j}}{n_{j} \overline{\beta_{j}}}$. Il suffit donc de montrer pour chaque j l'inégalité $\frac{n_{j} \overline{\beta_{j}}}{m_{j}}>\frac{n_{j+1} \overline{\beta_{j+1}}}{m_{j+1}}$. D'après [Za1], Chap.II, 3.11 , on a l'égalité

$$
n_{j+1} \overline{\beta_{j+1}}=n_{j+1} n_{j} \overline{\beta_{j}}+\left(m_{j+1}-n_{j+1} m_{j}\right) l_{j}
$$

et l'inégalité annoncée est alors conséquence de l'inégalité $n_{j+1} m_{j}<m_{j+1}$ et de l'inégalité $l_{j} m_{j}=\beta_{j}<\overline{\beta_{j}}$ pour $j \geq 2$.

On peut remarquer que les $\rho_{j}$ sont plus petits que 1 , et que le plus petit d'entre eux est $\frac{1}{m}$. Par exemple, pour la courbe d'exposants de Puiseux $(3 / 2,7 / 4)$, on a $\rho_{1}=1 / 4, \rho_{2}=7 / 26$.

Proposition 3.5. (P. Popescu-Pampu) La connaissance des exposants $\rho_{j}$ et des nombres d'intersection en 0 de chaque paquet $\Gamma^{(j)}=\Gamma^{Q(j)}$ de la courbe polaire avec la branche $C$ détermine le type topologique de $C$. 
En effet, d'après [Me], le nombre d'intersection $\left(\Gamma^{(j)}, C\right)$ vaut $\sigma_{j}=\left(n_{j}-1\right) \bar{\beta}_{j}$, et d'après ce que nous venons de voir, $\rho_{j} \sigma_{j}=\frac{\left(n_{j}-1\right) m_{j}}{n_{j}}$. Mais par construction nous avons $\operatorname{pgcd}\left(m_{j}, n_{j}\right)=1$, $\operatorname{donc} \operatorname{pgcd}\left(\left(n_{j}-1\right) m_{j}, n_{j}\right)=1$, et pour obtenir $m_{j}$ et $n_{j}$ il suffit de mettre $\rho_{j} \sigma_{j}$ sous forme irréductible. Les $\left(m_{j}, n_{j}\right)_{1 \leq j \leq g}$ sont alors déterminés, et donc la multiplicité $m=\prod_{j=1}^{g} n_{j}$ et les $\beta_{j}$.

Géométriquement, le nombre $\left(n_{j}-1\right) \bar{\beta}_{j}$ s'interprète comme le nombre des points de $C(\lambda)_{\epsilon}$ qui sont contenus dans la réunion des boules $\bigcup_{q \in Q(j), l} \mathbf{B}_{\Gamma_{q}, l}^{Q(j)}$ correspondant au sommet $Q(j)$; c'est aussi le nombre des boules, comptées avec multiplicité, de rayon $|\lambda|^{\rho_{j}}$, c'est à dire enfin la partie de la courbure de $C(\lambda)_{\epsilon}$ qui se concentre à l'échelle $\rho_{j}$. En fait, on a:

$$
\lim _{\lambda, \epsilon \rightarrow 0,|\lambda| \ll \epsilon} \int_{C(\lambda)_{\epsilon} \cap\left(\bigcup_{q \in Q(j), l} B_{\Gamma_{q}, l}^{Q(j)}(\lambda)\right)}|K| d v=2 \pi\left(n_{j}-1\right) \bar{\beta}_{j} .
$$

En résumé, dans le cas d'une branche, si l'on connaît les $\rho_{j}$ et la partie de la courbure qui se concentre à l'échelle $\rho_{j}$, on connaît le type topologique de la singularité limite.

Le cas d'une courbe réductible est plus compliqué et nous renvoyons à [GB2] pour des résultats utiles.

\section{Structure des boules $B_{q, l}^{Q}(\lambda)$}

Le but de cette section est d'éclaircir la disposition des boules $\left\{B_{q, l}^{Q}\right\}_{Q, q}$ quand $Q$ varie dans l'ensemble des sommets noirs du diagramme d'Eggers $T(C)$ et $P_{q}$ dans celui des branches du paquet $\Gamma^{Q}$.

Remarquons d'abord que les boules correspondant aux branches d'un même paquet ne sont pas nécessairement distinctes.

\section{Proposition 4.1.}

1. Si $Q$ est un sommet noir de $T(C)$ tel que $\rho(Q)>\frac{1}{\epsilon(Q)}$, alors les boules associées à une branche fixée $P_{q}(\tau)$ du paquet $\Gamma^{Q}(\tau)$ sont disjointes quand $\lambda$ est assez petit.

2. Soient $Q$ et $Q^{\prime}$ deux sommets noirs différents sur la même chaîne élémentaire de $T(C)$ tels que $\rho(Q) \neq \rho\left(Q^{\prime}\right)$. Si $P_{q}$ (resp. $P_{q^{\prime}}$ ) est une branche du paquet $\Gamma^{Q}$ (resp. $\left.\Gamma^{Q^{\prime}}\right)$, alors aucune boule associée à la branche $P_{q}$ ne rencontre une boule associée à la branche $P_{q^{\prime}}$ quand $\lambda$ est assez petit.

Démonstration

Soit $\left\{c_{i}=\left(x_{i}, y_{i}\right)\right\}_{i=1}^{\left(C, P_{q}\right)}$ l'ensemble des centres des boules $B_{q, l}^{Q}$ associées à la 
branche $P_{q}$ du paquet $\Gamma^{Q}$. On sait d'après la définition des boules $B_{q, l}^{Q}$ que

$$
x_{i}=c\left(\omega^{l_{i}} \lambda\right)^{\frac{m_{q}}{e_{q}+m_{q}}}+\cdots,
$$

où $c \neq 0, \omega$ est une racine primitive $\left(e_{q}+m_{q}\right)$-ième de l'unité et $l_{i} \in\left\{0, \ldots, e_{q}+\right.$ $\left.m_{q}-1\right\}$. Si on prend deux centres $c_{i}$ et $c_{j}$ différents on a:

$$
x_{i}-x_{j}=c(\lambda)^{\frac{m_{q}}{e_{q}+m_{q}}} \underbrace{\left[\omega^{\frac{l_{i} m_{q}}{e_{q}+m_{q}}}-\omega^{\frac{l_{l} m_{q}}{e_{q}+m_{q}}}\right]}_{\alpha}+\cdots
$$

où $c$ et $\alpha$ sont non nuls ( $\mathrm{si}$ on suppose $\alpha=0$ alors $\left(l_{i}-l_{j}\right) m_{q}>\left(e_{q}+m_{q}\right)^{2}$ ce qui est une contradiction car $l_{i}-l_{j}$ et $m_{q}$ sont plus petits que $\left.e_{q}+m_{q}\right)$.

Ainsi $\rho(Q)>\frac{1}{\epsilon(Q)}=\frac{m_{q}}{e_{q}+m_{q}}$ et $|\lambda|^{\rho(Q)}<|\lambda|^{\frac{1}{\epsilon(Q)}}$ quand $\lambda$ tend vers 0 . Donc la distance entre les centres $c_{i}$ et $c_{j}$ est plus grande que le rayon des boules et les boules $B_{q, l_{i}}^{Q}$ et $B_{q, l_{j}}^{Q}$ sont disjointes quand $\lambda$ est assez petit.

Soit maintenant $c=(x, y)$ (resp. $\left.c^{\prime}=\left(x^{\prime}, y^{\prime}\right)\right)$ le centre d'une boule associée à la branche $P_{q}$ (resp. $\left.P_{q^{\prime}}\right)$, on a (remarque 3.1$)$

$$
\begin{gathered}
x=a \lambda^{\frac{m_{q}}{e_{q}+m_{q}}}+\cdots=a \lambda^{\frac{1}{\epsilon(Q)}}+\cdots \\
x^{\prime}=b \lambda^{\frac{m_{q^{\prime}}}{e_{q^{\prime}}+m_{q^{\prime}}}}+\cdots=b \lambda^{\frac{1}{\epsilon\left(Q^{\prime}\right)}}+\cdots
\end{gathered}
$$

où $a$ et $b$ sont non nuls.

Comme $Q$ et $Q^{\prime}$ sont sur la même chaîne élémentaire de $T(C)$ d'après le corollaire 6.3 de [GB2], quitte à permuter $Q$ et $Q^{\prime}$, on a $v(Q)<v\left(Q^{\prime}\right)$ et $\epsilon(Q)<$ $\epsilon\left(Q^{\prime}\right)$ donc

$$
x-x^{\prime}=b \lambda^{\frac{m\left(P_{q^{\prime}}(\tau)\right)}{\left(C, P_{q^{\prime}}(\tau)\right)}}+\cdots \quad \text { et } \quad\left\|x-x^{\prime}\right\| \sim|\lambda|^{\frac{m\left(P_{q^{\prime}}(\tau)\right)}{\left(C, P_{q^{\prime}}(\tau)\right)}} .
$$

Mais $\rho(Q) \geq \frac{1}{\epsilon(Q)}>\frac{1}{\epsilon\left(Q^{\prime}\right)}$ et $\rho\left(Q^{\prime}\right)>\frac{1}{\epsilon\left(Q^{\prime}\right)}$ car $v\left(Q^{\prime}\right)>1$ et $Q^{\prime}$ ne peut pas être le point base de $T(C)$ puisque $v(Q)<v\left(Q^{\prime}\right)$.

Ainsi $\max \left\{|\lambda|^{\rho(Q)},|\lambda|^{\rho\left(Q^{\prime}\right)}\right\}<\left\|x-x^{\prime}\right\|$ et quand $\lambda$ est assez petit les boules $B\left(c,|\lambda|^{\rho(Q)}\right)$ et $B\left(c^{\prime},|\lambda|^{\rho\left(Q^{\prime}\right)}\right)$ sont disjointes. 


\section{References}

[Ca1] E. Casas-Alvero, Infinitely near imposed singularities and singularities of polar curves, Mathematische Annalen 287 (1990).

[Ca2] E. Casas-Alvero, Base points of polar curves, Ann. Inst. Fourier 41 (1991).

[E] H. Eggers, Polarinvarianten und die Topologie von Kurvensingularitaen, Bonner Mathematische Schriften 147 (1983).

[F] W. Fenchel, On total curvature of riemannian manifolds I, J. London Math. Soc. 15 (1940), 15-22.

[GB1] E. García Barroso, Invariants des singularités de courbes planes et courbure des fibres de Milnor, Thèse, Université de La Laguna, Tenerife (Espagne), LMENS-96-35, ENS, 1996.

[GB2] E. García Barroso, Sur les courbes polaires d'une courbe plane réduite, soumis.

[La] R. Langevin, Courbure et singularités complexes, Comment. Math. Helv. 54 (1979).

[Me] M. Merle, Invariants polaires des courbes planes, Inv. Math. 41 (1977), 103-111.

[Ro] P. Rouillé, Thèse, Dijon, 1997.

[S] H. J. S. Smith, On the higher singularities of plane curves, Proc. London Math. Soc., Vol. VI, 1875, pp. 153-182.

[Te1] B. Teissier, Introduction to equisingularity problems, Proceedings of Symposia in Pure Mathematics, Algebraic Geometry, Arcata 1974, Vol. 29.

[Te2] B. Teissier, The hunting of invariants in the geometry of discriminants, Real and complex singularities, Oslo 1976, Proceedings of the Nordic Summer School, Oslo, 1976.

[Te3] B. Teissier, Variétés polaires. I. Invariants polaires des singularités des hypersurfaces, Inv. Math. 40 (1977).

[Te4] B. Teissier, Courbes polaires relatives et courbure d'hypersurfaces de niveau, manuscrit, 1988, non publié.

[Za1] O. Zariski, Le problème des modules pour les branches planes, Centre de Mathématiques de l'École Polytechnique, 1973. Reédition Hermann, 1986.

[Za2] O. Zariski, General theory of saturation and of saturated local rings II, Equisingularity on Algebraic Varieties, Collected Papers, Volume IV.

[Za3] O. Zariski, Questions in Algebraic varieties, Contribution to the problems of equisingularity, CIME, Edizioni Cremonese, Roma 1970, pp. 261-343.

Evelia García Barroso

Dpto de Matemática Fundamental

Facultad de Matemáticas

Universidad de La Laguna

38271, La Laguna, Tenerife

Espagne

e-mail: ergarcia@ull.es
Bernard Teissier

D.M.I. Ecole normale supérieure

U.R.A. No. 762 du C.N.R.S.

45 Rue d'Ulm

F-75005 Paris

France

e-mail: teissier@dmi.ens.fr

(Received: May 29, 1998) 